\title{
Arbor
}

\section{Colaboración internacional en la industria aeroespacial de defensa}

Fernando Mosquera Silvén

Arbor CLXXI, 674 (Febrero 2002), 457-482 pp.

En este artículo el lector podrá conocer de forma clara las razones que han llevado a dicha colaboración en Europa, su gestación y programas en curso y futuros.

Respecto a los programas en curso, se tratan en profundidad aquellos en los que España está participando, como el Avión de Combate Europeo (Eurofighter 2000), el Avión de Transporte Militar Europeo (A-400M), el Misil Aire-Aire de guía radar activa y alcance superior al visual (Meteor) o el satélite de Observación Helios.

Sobre los programas futuros, hay que decir que el autor trata aquellos en que el Ejército del Aire está interesado, como:

- El misil Infrarrojo Aire-Aire de corto alcance Iris-T

- El Entrenamiento Avanzado Europeo de Pilotos de Combate (AEPJT)

- El designador de blancos electro-óptico de $4^{a}$ generación

- El Programa Europeo de Desarrollo Tecnológico (EATP)...

\section{Introducción}

El Siglo XX, el último del pasado Milenio, vio el nacimiento y el desarrollo espectacular de la aviación primero y, posteriormente, de los medios espaciales. 
Partiendo de unos principios modestos y elementales, durante el transcurso del siglo se produjo un desarrollo extraordinario y sorprendente de los medios aéreos, parejo al de la tecnología de cuyo progreso dependía a la vez que la estimulaba, para avanzar más y más y así poder llegar cada vez más lejos, más alto, más rápido, con mayor capacidad y energía de maniobra. Estos desarrollos hacen que el siglo XX bien pueda llamarse el siglo de la tecnología y de la aviación.

Este desarrollo de la aviación ha permitido a su vez el incremento continuo de las capacidades de los medios de que están dotadas las Fuerzas Aéreas, en particular el Ejército del Aire. Pero la complejidad tecnológica de estos medios, y el incremento inherente en el coste de los mismos, ha tenido como consecuencia que, con la excepción de los Estados Unidos, sea muy difícil que hoy en día una sola nación pueda desarrollar por sí misma un moderno avión de combate o de transporte estratégico. Por ello, las industrias aeroespaciales por una parte y los gobiernos de las naciones por otra, han ido constituyendo alianzas y uniones las primeras y estableciendo acuerdos de colaboración las segundas, de manera que puedan afrontar tecnológicamente con éxito y a un coste permisible el desarrollo de los medios aeroespaciales requeridos por las necesidades de la seguridad y defensa nacionales.

\section{Acuerdos de colaboración de los Gobiernos Europeos en relación con la industria de defensa}

El elevado coste del desarrollo tecnológico, principalmente de los medios aeroespaciales, que desborda las posibilidades individuales de las naciones, ha hecho que la cooperación en materia de seguridad se convierta en una necesidad. Así lo han entendido los Gobiernos Europeos a partir del final de la Segunda Guerra Mundial que, de manera ocasional y mediante colaboraciones limitadas a dos o tres países inicialmente y de forma decidida y continuada después, han llevado a cabo la realización de proyectos multinacionales cuya financiación y consecución hubiera sido irrealizable para las naciones abordadas aisladamente.

Ejemplos de estas primeras colaboraciones, en la década de los 60, fueron entre otros los programas para el desarrollo y producción de los aviones de transporte Transall, de patrulla marítima Breguet Atlantic y de combate Jaguar y Fiat G91.

Estas colaboraciones se han ido incrementando y ampliando con el paso del tiempo, a medida que se intensificaba el progreso y la complejidad tecnológica junto con la reducción de los presupuestos de Defensa, no 
Colaboración internacional en la industria aeroespacial...

sólo en lo que se refiere a la iniciación de proyectos comunes sino también al establecimiento de acuerdos internacionales que favorecieran la materialización de esos acuerdos en proyectos de desarrollo concretos.

Uno de los foros establecidos para facilitar la consecución de acuerdos que permitan el inicio conjunto de programas de desarrollo aeroespaciales en el área de la Defensa es la Conferencia de Directores Nacionales de Armamento de la OTAN. Cuando un país tiene necesidad de iniciar el desarrollo de un nuevo Sistema de Armas, puede proponer a los restantes miembros de esta Organización la participación en el nuevo desarrollo para conseguir repartirse los costes de desarrollo del mismo y reducir los de producción y apoyo logístico posteriores, consiguiendo a la vez incrementar la interoperabilidad entre las Fuerzas Aéreas de los países participantes en este nuevo proyecto. Este fue, por ejemplo, el caso del Programa para el desarrollo y producción del avión de combate Tornado.

En 1967 tres naciones de la OTAN, Alemania, Gran Bretaña e Italia, necesitaban modernizar sus Fuerzas Aéreas pero no podían permitirse el llevar a cabo programas nacionales separados para el desarrollo del nuevo avión de combate que necesitaban para reemplazar, en el caso de Alemania e Italia, los aviones F-104 Starfighter y, por parte de Gran Bretaña, los Vulcan, Canberra y Buccaneer. Antes de comprometerse a un programa de colaboración las tres naciones llevaron a cabo estudios conceptuales, llegando a la finalización de los mismos a la conclusión de que los diferentes requisitos de las tres naciones eran reconciliables y que el diseño y desarrollo de un único avión de combate "multi-role» (MRCA) para satisfacer sus necesidades era viable. En consecuencia, en 1969 firmaron un Memorandum de Entendimiento para colaborar en el nuevo proyecto que se denominó «MRCA». En base a este proyecto se inició en 1970 el desarrollo del nuevo avión, que recibió el apelativo de «Tornado», para lo cual se creó una Agencia OTAN encargada de la gestión del Programa, la Agencia NAMMA (NATO Multi-role combat aircraft Management Agency). Posteriormente, en 1976, se inició la producción de un total de 977 aviones para el conjunto de las tres naciones.

En base a la experiencia adquirida en el Programa Tornado, en 1985 Alemania, España, Gran Bretaña e Italia, decidieron iniciar un programa de colaboración para el desarrollo de un nuevo avión de combate europeo, programa denominado inicialmente EFA (European Fighter Aircraft), que pasó a denominarse posteriormente Eurofighter 2000. Pero este programa lo expondremos con más detalle posteriormente.

Aparte del foro existente en el seno de la OTAN para la colaboración en materia de armamentos, en 1976, los Ministros de Defensa de las naciones Europeas de la OTAN, excepto Islandia, -Alemania, Bélgica, Di- 
namarca, España, Francia, Gran Bretaña, Grecia, Holanda, Italia, Luxemburgo, Noruega, Portugal y Turquía- establecieron entre estos 13 países un foro para la cooperación en materia de armamentos, el Grupo Europeo Independiente de Programas (Independent European Programme Group -IEPG-). Fue en el marco de este foro donde se identificaron, en 1985, las necesidades futuras de un sistema de transporte aéreo militar destinado a satisfacer las necesidades de los países participantes más allá del año 2000, de donde nació el Programa del Avión de Transporte Militar Europeo, A400M, del que hablaremos más adelante.

Posteriormente, la Unión Europea Occidental, en su declaración con motivo de la firma del Tratado de Maastricht en 1991, propuso que se estudiara la posibilidad de incrementar la cooperación en materia de armamentos con el objetivo de crear una Agencia Europea de Armamentos con la doble finalidad de conseguir una mayor comunalidad e interoperabilidad entre las Fuerzas Armadas de los países europeos y el desarrollo de una industria europea de la defensa fuerte, con una buena base tecnológica, capaz de competir en los mercados internacionales, principalmente frente a los Estados Unidos. Como primer paso, los Ministros de Defensa de los 13 países del Grupo Europeo Independiente de Programas (IEPG), en su reunión en Bonn en diciembre de 1992, decidieron transferir las funciones del IEPG a la Unión Europea Occidental para de esta manera contar con un único foro de cooperación en materia de armamentos en Europa, que sería gestionado por los Directores Nacionales de Armamento de las 13 naciones, con los mismos derechos y responsabilidades cada uno de ellos, respondiendo ante sus Ministros de Defensa. A partir de la reunión de Ministros de Defensa de la Unión Europea Occidental en Roma, en mayo de 1993, este foro se denominó Grupo de Armamentos de la Europa Occidental (Western European Armaments Group -WEAG-), que tenía el objetivo de utilizar mejor los recursos de los países participantes mediante una armonización de sus requisitos, el fortalecimiento de la base industrial y tecnológica Europea y la cooperación en investigación y desarrollo. Como consecuencia de la creación de este nuevo foro, el IEPG fue absorbido por la nueva organización.

Más tarde, en noviembre de 1996, se aprobó por los Ministros de la Unión Europea la creación de la Organización de Armamentos de la Europa Occidental (Western European Armaments Organization -WEAO-), limitada inicialmente a establecer contratos en programas de investigación y desarrollo relacionados con la defensa en nombre de las naciones pertenecientes al WEAG.

La WEAO se puede considerar como la precursora de la Agencia Europea de Armamentos (European Armaments Agency -EAA-) que se pre- 
Colaboración internacional en la industria aeroespacial...

veía crear en base a los acuerdos del Tratado de Maastricht pero, como se acaba de decir, al quedar inicialmente limitada su actividad a los programas de investigación y desarrollo, y para acelerar el establecimiento de una Agencia que cubriera la gestión de toda clase de armamentos, los Ministros de Defensa de Alemania, Francia, Gran Bretaña e Italia firmaron en Estrasburgo, el 12 de noviembre de 1996, una declaración para la creación de una nueva Agencia de cooperación en materia de armamentos denominada «Organización Conjunta para la Cooperación en materia de Armamentos -OCCAR-», que fue formalmente establecida en enero de 1997, con sede en Bonn, para actuar como una oficina de programas conjunta en nombre de los países participantes. Entre los objetivos que se fijaban para la Organización estaban la coordinación de los requisitos a largo plazo de las naciones participantes, una política común de inversiones en el sector tecnológico de la defensa, mejorar la competencia y la explotación selectiva de la industria desarrollando una relación más estrecha entre las empresas de los países y su complementariedad y la renuncia al reparto de trabajos calculado en base a las adquisiciones realizadas por cada país en cada programa individual, aceptando a cambio que este cálculo se haga de forma global a lo largo de los años y de varios programas.

La OCCAR tiene carácter de organización abierta, siendo necesario para incorporarse a la misma solicitar el ingreso con la participación en uno de los programas que gestione la Organización y firmando la Convención que rige su actuación y que tiene carácter de tratado internacional. Actualmente han solicitado su incorporación España, Bélgica y Holanda. España y Bélgica a través del Programa del Avión de Transporte Europeo -A400M-, y Holanda con el del helicóptero NH-90.

Como un paso más en este espíritu de cooperación europea en materia de armamentos, los Ministros de Defensa de Alemania, España, Francia, Gran Bretaña, Italia, se reunieron en París el 20 de abril de 1998 para discutir intereses comunes en el área de la defensa y de la reestructuración de la industria de defensa, como consecuencia de la cual emitieron una declaración que fue respaldada asimismo por Suecia y que condujo finalmente a la firma de una Carta de Intenciones (Letter Of Intent -LOI-), que fue firmada en Londres el 6 de julio de 1998 por los Ministros de Defensa de estos seis países, y cuyo objetivo es establecer un marco cooperativo para facilitar la reestructuración de la industria de defensa europea, buscando su competitividad y eficacia, obteniendo sinergias operativas a través de los diferentes sectores de negocios, así como la eliminación de la duplicación de capacidades estableciendo una distribución óptima de las mismas (Centros de Excelencia). 
Por otra parte, dado que, como se ha dicho anteriormente, uno de los objetivos buscados con estos acuerdos de cooperación es incrementar la interoperabilidad entre las Fuerzas Armadas de los países participantes armonizando sus requisitos operativos, existe otro foro Europeo donde se reúnen los Jefes de Estado Mayor de las Fuerzas Aéreas de los países de la OTAN y de otros países no pertenecientes a esta organización, que son los responsables de aprobar los correspondientes requisitos operativos de sus Fuerzas Aéreas respectivas. Este foro se denomina EURAC (European Air Chiefs), que se estableció en 1993 en París con el objetivo de fomentar la cooperación y la amistad entre las distintas Fuerzas Aéreas Europeas y mejorar la eficacia e interoperabilidad de éstas por medio de la cooperación en el nuevo entorno europeo de seguridad y defensa.

\section{Participación española en programas aeroespaciales de colaboración europea}

Inicialmente el Ejército del Aire, creado en 1939, se dotó de material fabricado en España, aunque de diseño alemán en su mayoría, como eran el Messerschmitt 109, el Heinkel 111, el Junker 52, y la avioneta de enseñanza Bücker, contribuyendo de esta manera a permitir el desarrollo inicial de la industria aeronáutica nacional.

A partir de este primer impulso la empresa Construcciones Aeronáuticas S.A.-CASA-, desarrolló sus primeros proyectos propios como fueron los aviones de transporte Alcotán y Azor y, por su parte, la empresa Hispano Aviación acometía los proyectos de los aviones Triana, Saeta y HA300 , estos dos últimos reactores desarrollados con la cooperación del fabricante alemán Messerschmitt.

Sin embargo, en la década de los años 50, al establecerse los primeros Convenios de Ayuda con los Estados Unidos, que tenían un importante contenido de ayuda militar, la industria aeronáutica nacional sufrió un importante retroceso quedando prácticamente limitada a tareas de mantenimiento de aviones de procedencia norteamericana.

Afortunadamente, en la década siguiente, se inicia un Programa de inversiones para la modernización de las Fuerzas Armadas. En el marco de este Programa, el Ejército del Aire contrató con CASA, bajo licencia de la compañía americana Northrop, la fabricación de los aviones reactores de enseñanza y ataque F-5, que supuso para la industria aeronáutica española la incorporación de maquinaria, tecnología y procesos productivos modernos, esenciales para su nuevo despegue como industria aeronáutica que se confirmó con la iniciación de un nuevo proyecto de diseño y de- 
sarrollo de un avión de transporte ligero español, el CASA-212-Aviocar, que realizó su primer vuelo en 1971, y que ha tenido un gran éxito de ventas a nivel mundial; siguiéndole posteriormente el CASA-Nurtanio235 y el CASA-295, formando los tres una familia de aviones de transporte tácticos sin rival en el mundo occidental.

En la década de los 70, el Ejército del Aire inicia una política de diversificación de fuentes de suministro, adquiriendo en Francia primero los aviones Mirage III y, posteriormente los Mirage F-1, introduciendo a la vez el concepto de compensaciones industriales que obligaban a los vendedores a transferir tecnología de defensa a las industrias españolas, permitiendo así que éstas pudieran reducir en alguna medida su retraso tecnológico con respecto a las europeas. Esta política de transferencia de tecnología se intensificó todavía más con la adquisición posterior, en la década de los 80, a los Estados Unidos, de los aviones de combate F-18, unos de los más avanzados de la época, que permitió a la industria nacional, tanto de fabricación de aviones como de electrónica de defensa, dar un paso trascendente que le ha permitido participar con éxito en el desarrollo del avión de combate europeo, el Eurofighter 2000, al mismo nivel que las industrias de los otros tres países participantes, Alemania, Gran Bretaña e Italia, así como en el resto de los programas que vamos a exponer a continuación.

\section{Programas actualmente el desarrollo}

\section{Avión de combate Europeo -Eurofighter 2000-}

En 1977, los Ministros de Defensa de Alemania, Gran Bretaña y Francia, en el seno del Grupo Europeo Independiente de Programas (IEPG), identificaron conjuntamente la necesidad operativa de un nuevo avión de combate, al que se denominó ACA (Agile Combat Aircraft). Posteriormente se unió Italia y, más tarde, en 1983, España, iniciándose un estudio conjunto de viabilidad para el desarrollo de un avión que satisficiera los requisitos previstos de un avión de superioridad aérea de altas características para el comienzo del siglo XXI. De estos estudios se dedujo que existían importantes diferencias entre los requisitos franceses y los de las otras cuatro naciones que no hacían posible una solución común por lo que, en agosto de 1985, por el Acuerdo de Turín, Alemania, España, Gran Bretaña e Italia decidieron iniciar un programa de colaboración que se denominó EFA (European Fighter Aircraft), y más adelante Eurofighter, retirándose Francia para desarrollar por sí sola el avión Rafale. 
Los objetivos comunes de las cuatro naciones para el Programa EFA eran los siguientes:

- Proporcionar un Sistema de Armas aéreo que satisficiera los requisitos operativos definidos por las cuatro naciones. El «role» primario del nuevo avión sería el de Aire-Aire, teniendo asimismo la capacidad Aire-Tierra como «role» secundario.

- Producir el nuevo avión en los plazos requeridos.

- Minimizar los costes totales y compartir los costes y el trabajo del proyecto en relación con el número de aviones a adquirir por cada nación.

A estos objetivos comunes hay que añadir los que quería alcanzar España por su parte, que eran los siguientes:

- Mantener un adecuado nivel de desarrollo tecnológico de la industria aeronáutica española, disminuyendo o eliminando las diferencias con el resto de Europa.

- Disponer del conocimiento y control de la tecnología empleada.

- Aumentar el grado de independencia estratégica.

- Adquirir experiencia en la participación en programas plurinacionales.

En octubre de 1986 se firmó en Escocia, por los Ministros de Defensa de los cuatro países participantes, el Memorandum de Entendimiento Número Uno, o Memorandum General, que regiría el Programa a lo largo de todas sus fases. Las fases que se consideraron para el Programa son las siguientes:

- Fase de Definición.

- Fase de Desarrollo.

- Fase de Inversiones para la Producción y de Producción.

- Fase Operativa.

Cada una de estas fases estaría cubierta por su Memorandum de Entendimiento correspondiente por el cual se comprometen las naciones a la financiación y a llevar a cabo los trabajos de la fase correspondiente, firmándose el Número Dos, correspondiente a la Fase de Definición, en noviembre de 1986.

En febrero de 1987 se decide que el Programa se transfiera desde el IEPG, donde se había originado, a la OTAN, creándose una Agencia OTAN para la gestión del Programa, la Agencia NEFMA (NATO European Fighter Aircraft Management Agency), cuyo personal pertenece a las cuatro naciones participantes en el Programa.

Finalizada la Fase de Definición del Proyecto, en noviembre de 1988 se firma el Memorandum de Entendimiento Número Tres, correspondiente a la siguiente Fase, la de Desarrollo. 
Colaboración internacional en la industria aeroespacial...

Para llevar a cabo el desarrollo del nuevo Sistema de Armas se establecieron dos Consorcios Industriales, uno como contratista principal del Sistema de Armas en general y otro del motor. El primero de ellos, denominado Eurofighter, estaba compuesto por las siguientes compañías aeronáuticas: DASA de Alemania, CASA de España, British Aerospace (BAE) de Gran Bretaña y Alenia de Italia. Por lo que se refiere al consorcio del motor, denominado Eurojet, estaba compuesto de las siguientes compañías: MTU de Alemania, ITP de España, Rolls Royce de Gran Bretaña y Fiat Aviazione de Italia. Cada una de estas compañías subcontrata a su vez el desarrollo y producción de los diferentes equipos del avión con una serie de empresas de las naciones del programa; en el caso de España están participando cerca de 20 empresas principales de los sectores aeroespacial, de electrónica, de armamento, de automoción y auxiliar y alrededor de otras 250 empresas subsidiarias.

En pleno proceso de desarrollo del nuevo Sistema de Armas tiene lugar un cambio del escenario geopolítico en Europa con el final de la guerra fría y la reunificación alemana. Como consecuencia de ello, las naciones del programa se plantean si el avión que se había definido con un requisito operativo apto para la situación geopolítica anterior era todavía el adecuado al nuevo y previsible futuro escenario, decidiéndose como consecuencia de ello llevar a cabo una revisión en profundidad de esos requisitos. Como resultado de la misma los Ministros de Defensa deciden, en la reunión que tuvo lugar en Bruselas en diciembre de 1992, llevar a cabo una reorientación del Programa que produjo cambios en las especificaciones operativas del Sistema de Armas, siendo uno de los más significativos el de dotarle de una mayor capacidad de ataque al suelo, equiparando así los «roles» Aire-Aire y Aire-Suelo, modificando asimismo los plazos de desarrollo del Programa que sufrieron un retraso importante. Estos acuerdos se materializaron en lo que se denominó Memorandum de Entendimiento para la Reorientación de la Fase de Desarrollo, el Número Cuatro, que se firmó en julio de 1995, cambiándose de nuevo el nombre del Programa, que pasó a denominarse Eurofighter 2000.

$\mathrm{Al}$ objeto de reducir los costes de gestión tanto del Programa Eurofighter como del Programa Tornado, las naciones participantes en los mismos, que eran las mismas para los dos excepto en el caso de España que sólo participaba en el Eurofighter, decidieron integrar las dos Agencias OTAN, NAMMA y NEFMA, en una sola que pasó a denominarse NETMA -NATO Eurofighter and Tornado Management Agency-, firmándose en octubre de 1995 un nuevo Memorandum de Entendimiento, el Número Cinco. 
Una vez que las naciones consideraron que el desarrollo del Sistema de Armas se podía considerar como maduro, aunque todavía no finalizado, decidieron aprobar el inicio de la siguiente Fase, la de Producción, lo que tuvo lugar mediante la firma por los Ministros de Defensa, en diciembre de 1997 en Bonn, de dos nuevos Memorandums de Entendimiento, los Números Seis y Siete, el primero para la Producción del avión y el segundo para el Apoyo Logístico al Sistema de Armas. Mediante ellos, las cuatro naciones del Programa se han comprometido a la adquisición de un total de 620 aviones, con su Apoyo Logístico correspondiente, distribuidos de la siguiente manera: 180 para Alemania, 87 para España, 232 para Gran Bretaña y 121 para Italia. Actualmente están volando siete prototipos, dentro de la fase de desarrollo, que han realizado ya más de 1.500 horas de vuelo, estando prevista la entrega de los primeros aviones a las Fuerzas Aéreas de los países participantes en el plazo de un año.

El nuevo avión de combate Europeo, Eurofighter 2000, también conocido por el apelativo de «Typhoon», está considerado por los especialistas como uno de los más avanzados de entre los que van a estar en servicio en las primeras décadas del presente siglo, comparable en muchos aspectos al considerado líder de esta categoría, el Estadounidense F-22, al que supera en el «role» Aire-Suelo, siendo algo inferior que él en el «role" Aire-Aire, principalmente por las extraordinarias características «stealth» de éste y su mayor potencia de motor.

En el avión se integran del orden de 280 equipos diferentes, el $80 \%$ de ellos de nuevo desarrollo como son, por ejemplo, algunos de los más esenciales como el radar, el sensor pasivo de infrarrojos, los equipos de guerra electrónica, etc.

A continuación vamos a describir algunas de las características destacadas del extraordinario Sistema de Armas que es el Eurofighter 2000 y de los equipos principales de que está dotado.

\section{Capacidad «supercruise»}

El Eurofighter tiene la posibilidad de volar a velocidad superior a la del sonido sin necesidad de utilizar el postquemador, es decir está dotado de capacidad «supercruise», característica que le proporciona una ventaja importante para el combate, así como en la reducción de consumo de combustible y de la señal infrarroja que produce la emisión de gases del motor. 
Fusión de sensores

Esta es una capacidad que en el Eurofighter está extraordinariamente desarrollada y se puede considerar perfectamente comparable a la del F-22 americano.

Los diferentes sensores del Eurofighter proporcionan datos procedentes del radar, del sensor pasivo de infrarrojos «Infrared Search And Track» (IRST), del identificador amigo/enemigo (IFF), de las medidas de apoyo electrónico (ESM) y, vía enlace de datos, de fuentes externas, mezclándolos y fusionándolos presentándole al piloto, finalmente, una única representación táctica, siendo la integración de los sistemas automática, así como la identificación y priorización de las amenazas, proporcionándole así al piloto solamente la información que necesita y cuando la necesita, depurada automáticamente para permitirle concentrarse en los aspectos tácticos de la misión.

El proceso de fusión de sensores produce una única traza de cada blanco individual que puede ser reportado por varios sensores simultáneamente, cada uno proporcionando un subconjunto de atributos que se compilan para producir una visión más completa del blanco. Los algoritmos del software de los ordenadores del avión miden la fiabilidad de cada información antes de combinarlos para producir una identidad y prioridad fusionada del blanco. El aspecto, color y contenido del símbolo de cada blanco, le dice al piloto de qué sensor provienen los datos.

\section{Extraordinaria maniobrabilidad}

Ésta es otra característica en la que el Eurofighter puede no solamente ser considerado como comparable al F-22 americano, sino incluso aventajarle en algunos aspectos. El Eurofighter es un caza polivalente, bimotor, extraordinariamente ágil gracias a su configuración delta/canard que le hace considerablemente inestable y le proporciona gran agilidad, con una baja carga alar, un «drag» mínimo y una gran relación potencia / peso. Optimizado para el combate aéreo, tanto en distancias más allá del alcance visual como para el combate cerrado.

La configuración inestable delta/canard combina un bajo drag supersónico con una gran agilidad subsónica. La baja carga alar y la alta relación potencia/peso combinadas proporcionan una gran maniobrabilidad, así como capacidad para despegues cortos.

El combate más allá del alcance visual requiere una gran aceleración y régimen de subida para proporcionar la máxima energía, y por lo tan- 
to alcance, de lanzamiento de los misiles. Para maniobrar en la posición de ataque sin perder esa energía se requiere un alto régimen sostenido de viraje supersónico, mientras que para escapar maniobrando de manera que se reduzca el alcance efectivo de los misiles del enemigo se requiere un alto régimen instantáneo de viraje. El combate cerrado requiere gran agilidad. La configuración delta/canard ofrece la mejor combinación de gran agilidad y pequeño tamaño.

Una configuración delta por sí sola no proporciona la agilidad requerida, pero los alerones delanteros («canard») ayudan a desestabilizar el avión. Por otra parte, la configuración inestable requiere la utilización de mandos electrónicos (fly-by-wire) para el control del avión debido a la nolinealidad de las ecuaciones aerodinámicas y para estabilizar artificialmente el avión. Para su control totalmente seguro, el avión dispone de un sistema de control de vuelo digital (Flight Control System) automatizado y cuadruplicado redundante.

\section{Capacidad «Stealth»}

Es preciso reconocer que el Eurofighter no es lo que se denomina un avión "stealth» (de baja observabilidad para cualquier sensor, especialmente los de tipo electrónico) de última generación y, por lo tanto, es en esta área donde el F-22 americano le aventaja de manera significativa.

De cualquier manera, el Eurofighter presenta algunos aspectos que contribuyen a la baja observabilidad de su diseño. Por una parte, su pequeño tamaño contribuye a reducir la posibilidad de detección por medios ópticos e incluso, en alguna medida, por los de tipo electromagnético como es el radar.

En lo que concierne a la detección por medio de radares, el avión se ha diseñado para cumplir el requisito establecido por las naciones de tener una Sección Cruzada Radar (RCS) frontal menor que la de cualquier avión en servicio o en producción cuando se establecieron dichos requisitos. A estos efectos hay que tener en cuenta que a la hora de aplicar las tecnologías «stealth» en los aviones de combate, las áreas consideradas más críticas son la frontal y posterior, por lo que es en estas áreas donde se requieren los niveles inferiores de firma radar.

Por otra parte, la filosofía del sistema permite la operación pasiva utilizando el sensor de infrarrojos de búsqueda y seguimiento, «Infrared Search And Track» (IRST), su capacidad de enlace de datos con el «Multifunctional Information Distribution System»(MIDS), que le permite conocer la situación de cualquier amenaza, ya sea aérea, naval o terrestre que le puedan proporcionar otras fuentes sin emitir ninguna señal 
Colaboración internacional en la industria aeroespacial...

por sí mismo, el casco de vuelo que le permite designar blancos a los misiles infrarrojos y otras capacidades que, en su conjunto, permiten al piloto del Eurofighter la posibilidad de detectar y seguir blancos aéreos y terrestres sin utilizar el radar, disminuyendo así sensiblemente la posibilidad de ser detectado.

Por lo que se refiere a la reducción de la señal infrarroja, el calor que generan los motores del Eurofighter, debido a su diseño, es sensiblemente inferior al de los aviones actuales.

Pasemos ahora a describir algunos de los equipos más destacados que incorpora el Eurofighter.

\section{Sensor pasivo de infrarrojos IRST/FLIR}

El equipo IRST/FLIR es uno de los que proporcionan al Eurofighter una capacidad adicional extraordinaria, superior a cualquiera de los aviones de caza en servicio actualmente. En su desarrollo y producción está participando una empresa española, Tecnobit.

El IRST/FLIR tiene dos modos, el IRST (Infrared Search and Track) y el FLIR (Forward Looking InfraRed). El más significativo de ellos es el primero, proporcionándole capacidades de detección similares a las del radar, aunque con un alcance algo menor, pero con la ventaja significativa de ser un detector pasivo, lo que evita su detección por el avión adversario colaborando así a su capacidad de no ser detectado («stealth»). Este modo IRST incluye las capacidades de seguimiento durante el barrido (Track While Scan -TWS-) de múltiples blancos, priorización automática de blancos y cálculo de distancia e identificación.

Por lo que se refiere al FLIR, constituye una ayuda secundaria para el aterrizaje y el vuelo a baja cota nocturno. Las imágenes obtenidas en el modo FLIR pueden ser representadas en la cabina para utilizarlas como ayudas a la navegación y al aterrizaje.

\section{MIDS}

El MIDS (Multifunctional Information Distribution System), es un equipo que confiere al avión una extraordinaria capacidad de supervivencia y supremacía en el escenario de la superioridad aérea. Consiste básicamente en un sistema de enlace de datos que permite la transmisión y recepción segura de información táctica entre el avión y/o los puestos de mando terrestres o aéreos, constituyendo una red de información 
y combate integrados en donde el conjunto de plataformas de combate forman una única plataforma virtual. De esta forma el avión puede recibir información de blancos adversarios que no puede detectar con sus propios sensores por encontrarse a distancias superiores al alcance de los mismos, o por debajo o detrás de él, dentro de la red de información de combate en que está integrado, ampliando así su capacidad de detección haciéndolo de una manera pasiva, lo que contribuye a su capacidad de no ser detectado («stealth»).

El Equipo MIDS ha sido desarrollado por un consorcio internacional en el que han participado Alemania, España, Estados Unidos, Francia e Italia. Por parte española la empresa líder es INDRA.

\section{Casco de vuelo}

El casco de vuelo del Eurofighter integra el sistema de navegación y ataque del avión, permitiendo la designación de objetivos y el apuntamiento de las armas, la presentación de datos en el propio visor del casco y la de imágenes vídeo procedentes del FLIR, ampliando así el ángulo de acceso a los datos de navegación y combate que proporciona el «Head Up Display -HUD-» de la parte frontal de la cabina, permitiendo al piloto mirar a través de la cúpula en cualquier dirección para seguir a los aviones adversarios durante las maniobras de combate. Incluye asimismo gafas de visión nocturna y protección láser.

En el visor del casco de vuelo se presentan los datos de navegación y los tácticos relativos al combate. En el caso de los misiles de corto alcance, su cabeza buscadora sigue el movimiento de la cabeza del piloto a través del casco de vuelo y se bloca en el blanco a decisión del piloto.

\section{DVI}

El DVI (Direct Voice Input) constituye una innovación en los aviones de combate. Permite al piloto conseguir con una simple orden verbal la mayoría de las cosas que puede hacer con sus dedos, con muy pocas excepciones como puede ser el lanzar las armas. Con el DVI se pueden, por ejemplo, entrar datos y llevar a cabo funciones que no afecten a la seguridad como selección de presentaciones en las pantallas, de canales de la radio y de blancos. Asimismo, el piloto jefe de una formación podrá asignar blancos a los otros miembros de la formación, a través de enlace de datos, con una orden dada por medio del DVI. 
Colaboración internacional en la industria aeroespacial...

Estas capacidades del DVI permiten aliviar la carga de trabajo del piloto en la cabina, lo que es esencial en un avión monoplaza, sobre todo en situaciones comprometidas en combate.

Finalmente, se puede decir que el Programa Eurofighter ha supuesto un importante paso adelante en la cooperación europea en materia de Defensa, así como para la industria aerospacial española que le ha servido para ponerse al nivel tecnológico del resto de industrias europeas.

\section{Avión de Transporte Militar Europeo -A400M-}

A principios de la década de los 80, en 1982, la industria aerospacial empezó a desarrollar ideas para el futuro avión de transporte militar. Participaban en este proyecto las compañías MBB de Alemania, Aerospatiale de Francia, British Aerospace de Gran Bretaña y Lockheed de Estados Unidos. El consorcio formado denominó al proyecto Futuro Avión Internacional de Transporte Militar, «Future International Military Airlifter -FIMA-». En 1987 la compañía española CASA y la italiana Alenia se unieron al consorcio FIMA y, dos años más tarde Lockheed decidió abandonarlo después de que el Gobierno americano confirmara sus planes de apoyar la producción del sucesor del Hércules, el C-130J.

En 1985, en el marco del Grupo Europeo Independiente de Programas (IEPG), se identificaron las necesidades futuras de un avión de transporte militar de capacidad media-alta destinado a satisfacer las necesidades a partir del año 2005 de los países participantes en el incipiente Programa -Alemania, Bélgica, España, Gran Bretaña, Francia e Italia -, con objeto de sustituir las flotas de aviones C-130 Hércules y C-160 Transall. En 1989 se adhiere al Programa Turquía, en 1991 Portugal y, finalmente, en 2000, Luxemburgo. El Programa recibió el nombre de Futuro Gran Avión, «Future Large Aircraft -FLA-».

Entre los años 1985 y 1991 se llevaron a cabo los estudios relativos a la armonización de las especificaciones operativas del nuevo Sistema de Armas que tenían en cuenta el nuevo contexto geoestratégico que requería, y requiere aún más en la situación actual, reforzar la capacidad de transporte militar, principalmente a larga distancia y con gran capacidad de carga, para asegurar la movilidad estratégica y poder transferir en plazos breves los efectivos de personal y los medios materiales necesarios para el despliegue principalmente de las Fuerzas de Intervención Rápida cuya actuación se determine como necesaria en cualquier tipo de crisis o conflicto. 
En 1991, con el objeto de llevar a cabo los estudios de previabilidad, se formó un consorcio industrial formado por las compañías europeas del anterior consorcio FIMA, denominándose el nuevo consorcio «European FLA Group -EUROFLAG-». Los estudios de previabilidad se iniciaron en enero de 1992 y fueron seguidos por los de viabilidad que dieron comienzo en noviembre de 1993, finalizando en diciembre de 1994.

A la recepción del resultado de los estudios anteriores las naciones por su parte elaboraron el documento Requisitos Europeos de Estado Mayor «European Staff Requirements -ESR-», que fue terminado en marzo de 1996 y firmado por los Jefes de Estado Mayor de las Fuerzas Aéreas de las naciones participantes.

En otoño del siguiente año las naciones del Programa envían al consorcio industrial una Petición de Oferta, a la que éste contesta, ya con el nuevo nombre de «Airbus Military Company -AMC-», dentro del plazo establecido, en enero de 1999. Sin embargo, en el intermedio, a instancias por una parte de Gran Bretaña, y por otra de Alemania, se solicitan en paralelo ofertas a la Compañía americana Lockheed Martín de su avión C130J, a Boeing del C-17, y a la rusa-ucraniana Antonov del An-70. Finalmente se decidió por las naciones del Programa que el avión que mejor satisfacía las necesidades de todas ellas era el A400M propuesto por AMC.

El Programa está próximo al inicio del desarrollo del avión después de que los Ministros de Defensa de las naciones participantes, excepto Italia, firmaran el correspondiente Memorandum de Entendimiento el pasado 18 de diciembre, con la autorización para la firma del contrato con AMC, aunque ésta no se llevará a cabo hasta la ratificación por el parlamento alemán de estas decisiones.

El Programa A400M, es el mayor programa de cooperación que ha existido hasta la fecha en lo que al número de naciones participantes se refiere, que aún así han sido capaces de acordar unos requisitos operativos comunes y el modo de llevar a cabo un programa esencial para cubrir las carencias en el área del transporte aéreo militar táctico y estratégico, carencias que se han puesto de manifiesto en los últimos conflictos como son los del Golfo Pérsico y de los Balcanes. Este nuevo sistema de transporte constituye un pilar básico en el desarrollo de la política Europea de Seguridad y Defensa con relación al despliegue de la Fuerza de Reacción Rápida y a la posible creación de un Mando de Transporte Aéreo Europeo.

En cuanto al desarrollo del programa se ha escogido una aproximación diferente a la del Programa Eurofighter, con el objeto de reducir riesgos y costes. El Programa se va a llevar a cabo en una fase única denominada Fase de Desarrollo y Producción, a ser gestionada de acuerdo con los principios de gestión industriales aprovechando la experiencia 
que tiene la compañía Airbus en el desarrollo de programas para la aviación comercial de transporte. Por lo que se refiere a las naciones, la gestión del Programa será realizada por la Agencia OCCAR, de la que se ha hecho mención en la primera parte de este artículo, creándose en el seno de la misma una Oficina específica del Programa en la que participará personal de todas las naciones implicadas.

Por lo que se refiere al reparto del trabajo, en lo que respecta a la estructura del avión y al motor se seguirá el mismo principio que en el Programa Eurofighter, cada nación tendrá derecho a una participación equivalente al número de aviones a adquirir, sin embargo, en los equipos que se integran en el avión se admite un poco más de flexibilidad para mejorar la relación coste-eficacia. Como dato significativo, a España le ha correspondido, entre otras actividades, la línea de montaje final de todos los aviones que se fabriquen, tanto de los 196 inicialmente contratados por las naciones -Alemania 73, Bélgica 7, España 27, Francia 50, Gran Bretaña 25, Luxemburgo 1, Portugal 3 y Turquía 10-, como los que se puedan exportar posteriormente a otras naciones, convirtiéndose así CASA en un Centro de Excelencia en lo que a la aviación de transporte militar se refiere.

En lo referente a la tecnología que incorpora el nuevo avión, se puede mencionar primero su diseño que ha sido realizado totalmente por medio de ordenador, siendo de destacar asimismo la utilización de estructuras ligeras y materiales que reducen el consumo de combustible aumentando en contrapartida el alcance; en particular, es la primera vez que se va a hacer la estructura completa del ala de un avión de transporte de material compuesto de fibra de carbono (Carbon Fiber Reinforced Plastic -CFRP-). Dispondrá de un avanzado sistema de control de vuelo, basado en los utilizados en los aviones civiles de Airbus así como en lo que se refiere al diseño de la cabina de pilotaje, con pantallas multifunción en color y aviónica digital integrada que permitirá reducir la carga de trabajo del piloto, estando preparada para la utilización de gafas de visión nocturna.

Misil Aire-Aire de guía radar activa y alcance superior al visual-Meteor-

Este Programa, denominado inicialmente FMRAAM (Future Medium Range Air to Air Missile), y más tarde (Beyond Visual Range Air to Air Missile -BVRAAM-), fue iniciado de manera independiente por Gran Bretaña, que redactó por sí sola los correspondientes Requisitos de Estado Mayor para el nuevo misil, enviando una Invitación para Ofertar a di- 
versas compañías europeas y estadounidenses en 1996. No obstante, dado el coste del proyecto, para poderlo compartir mediante un Programa de Colaboración Europeo con otras naciones, Gran Bretaña invitó primero en 1995 a Alemania, Italia y Suecia, y posteriormente, en 1996, a España, a participar en el Programa, invitación que fue aceptada así como los Requisitos de Estado Mayor elaborados por Gran Bretaña. Más tarde, Francia se uniría asimismo al Programa.

El objetivo del Programa era desarrollar un nuevo misil aire-aire de alcance superior al visual, capaz de operar eficazmente en los nuevos y futuros escenarios operativos, que tuviera unas características de combate muy superiores a las de los misiles en servicio, en particular al misil AMRAAM (Advanced Medium Range Air to Air Misil), en servicio en numerosas naciones.

Para cumplir lo anterior, era necesario actuar en varias áreas, una de las más importantes era la referente a la energía de propulsión del misil, tanto en su fase inicial de lanzamiento como, sobre todo, en su fase final, incrementando así su velocidad y maniobrabilidad. Prácticamente la mayoría de los misiles actuales de esta categoría disponen de un motor cohete de una sola etapa que permite al misil acelerar rápidamente hasta alcanzar su máxima velocidad en la primera parte de la trayectoria del misil; una vez que se extingue el motor el misil continúa su trayectoria hacia el blanco con una velocidad progresivamente menor, lo que tiene un efecto negativo secundario en el control de la dirección del misil ya que ésta se gobierna mediante las superficies externas de control del misil, actuadas por la presión aerodinámica, presión que se va reduciendo en paralelo con la reducción de la velocidad del misil, con lo que la eficacia de dichas superficies sufre una reducción equivalente, impidiendo que el misil pueda realizar virajes rápidos y de alta energía para poder seguir al blanco, lo que se considera esencial hoy día dado el incremento significativo que han alcanzado los aviones de combate modernos en su capacidad de maniobra a cualquier altura y velocidad, ya sea ésta subsónica o supersónica.

Para subsanar el problema anterior, la solución que se ha adoptado en el nuevo misil ha sido la de dotarle de un motor híbrido que integra un motor cohete y un motor estato-reactor accionado por la presión dinámica del aire.

Otras de las características mejoradas en el nuevo misil serán su capacidad de operar en ambiente severo de contramedidas electrónicas y su capacidad de enlace de datos para la dirección del misil en su fase inicial.

En el concurso industrial internacional que se llevó a cabo para el desarrollo del misil quedaron como finalistas la compañía americana Rayt- 
Colaboración internacional en la industria aeroespacial...

heon y un consorcio europeo formado por las principales industrias de misiles y electrónica de los países participantes, proponiendo estas últimas el misil Meteor, nombre con el que se ha bautizado definitivamente al Programa al ser esta la solución finalmente adoptada en base a las características técnicas y operativas del misil propuesto, solución que tiene la ventaja añadida de permitir establecer una alternativa europea a los misiles americanos de esta categoría, a lo que se añade la independencia operativa que proporciona.

El Programa ha sido aprobado recientemente por todas las naciones participantes excepto por Alemania, que se espera que lo haga en el transcurso del año 2002 permitiendo así proceder a la iniciación de la Fase de Desarrollo del misil.

A diferencia de los programas anteriores su gestión no se realizará por ninguna Agencia intergubernamental, haciéndose cargo de la misma la Agencia de Adquisiciones para la Defensa de Gran Bretaña, con participación de representantes de las naciones del Programa.

\section{Satélites de Observación - Helios -}

El Programa para el desarrollo de un Sistema de Satélites de Observación Militar, tiene por objeto satisfacer las necesidades de información estratégica de las Fuerzas Armadas de los países participantes, permitiendo disponer de información fiable, precisa y actualizada con independencia y discreción.

El Programa fue iniciado en solitario por Francia que, a la vista de los costes del mismo, decidió posteriormente hacerlo en colaboración con otras naciones invitando a Italia, que se adhirió en 1987, y a España, que lo hizo en 1988, firmándose en noviembre del mismo año un acuerdo entre estas naciones para llevarlo a cabo como un Programa de Colaboración.

La primera parte del Programa comprendía dos satélites, Helios I-A y I-B. El Helios I-A se lanzó al espacio en julio de 1995 desde el Centro Espacial Francés de Guayana, estableciéndose en una órbita baja (algo más de 600 Kilómetros) heliosíncrona que permite obtener imágenes en el espectro visible de cualquier punto de la Tierra. Por su parte, el Helios I-B se lanzó en diciembre de 1999, siendo de características similares al I-A, aunque con algunas mejoras en el área operativa, cuyo objetivo es dar continuidad al I-A cuando éste deje de estar operativo.

A los Helios I les sucederán otros dos satélites, Helios II-A, con lanzamiento previsto el año 2004, y Helios II-B, que incorporarán sensores 
tanto en el espectro visible como en el infrarrojo, mejorando asimismo las prestaciones de los Helios I en lo que se refiere a resolución y capacidad operativa. En esta segunda parte del Programa la participación de Italia ha sido sustituida por la de Bélgica.

Por lo que se refiere a la participación industrial en el programa, al igual que sucedía en los Programas de Colaboración que hemos visto anteriormente, en su desarrollo y producción participan las industrias de las naciones pertenecientes al Programa.

\section{Programas futuros}

\section{Misil infrarrojo Aire-Aire de corto alcance -IRIS-T-}

Con el objeto de desarrollar un nuevo misil aire-aire, infrarrojo, de corto alcance, que sustituyera a los actualmente en sèrvicio en numerosas naciones, principalmente los americanos de la familia Sidewinder, con unas prestaciones muy superiores a los mismos en lo que se refiere a agilidad de maniobra, capacidad de detección y capacidad de ser apuntado mediante el casco de vuelo del piloto, aprovechando así las innovaciones tecnológicas introducidas en los aviones de combate actuales, se inició un Programa de Colaboración Internacional liderado por Alemania y con la participación de Canadá, Grecia, Italia, Noruega y Suecia, mediante la firma de un Memorandum de Entendimiento en agosto de 1996.

A finales de 2001 Canadá se retiró del Programa, lo que ha permitido a España, que ha estado participando como observador en el mismo por tener la necesidad operativa de un misil de estas características, considerar la posibilidad de su incorporación, para lo cual se están llevando a cabo negociaciones que permitan determinar finalmente la conveniencia de integrarse en el Programa.

Para conseguir la agilidad de maniobra el misil estará dotado de una tobera vectorial. En cuanto al sensor infrarrojo de detección y búsqueda, incorporará la capacidad de comparación de imágenes para evitar ser confundido mediante el lanzamiento de bengalas por el avión adversario, teniendo un ángulo de detección de 180 grados.

La Fase de Producción del misil está prevista que se inicie en la segunda mitad del año 2002.

Al igual que en el Programa Meteor, la gestión del Programa no se realizará por ninguna Agencia intergubernamental, haciéndose cargo de la misma la Agencia de Adquisiciones para la Defensa de Alemania, con participación de representantes de las naciones del Programa. 
Colaboración internacional en la industria aeroespacial...

Entrenamiento Avanzado Europeo de Pilotos de Combate (Advanced European Jet Pilot Training -AEJPT-).

El Programa AEJPT tuvo su origen en 1997, en el seno de la Conferencia de Jefes de Estado Mayor del Aire Europeos (EURAC) mencionada en la primera parte de este artículo, teniendo como objetivo el desarrollo en colaboración, de un sistema Europeo para la formación de pilotos de combate, en un entrenador que les capacite para desarrollar posteriormente su actividad operativa en los aviones de combate de nueva generación, tales como el Eurofighter 2000, para lo que no son suficientes los aviones de entrenamiento actuales y no está justificado el desarrollo de una solución nacional individual, teniendo en cuenta el pequeño número de aviones de este tipo que necesitaría cada una de ellas.

Para realizar los estudios previos de viabilidad, la industria europea ha formado un grupo denominado «European Industry Working Group -EIWG-», en el que participan activamente compañías españolas del área aerospacial y de electrónica.

En base a los resultados obtenidos de los estudios de previabilidad, se ha redactado el Objetivo de Estado Mayor correspondiente y se ha firmado el pasado mes de diciembre un Memorandum de Entendimiento para la realización de la fase de viabilidad entre las naciones interesadas en este Programa que, inicialmente, son las siguientes: Alemania, Austria, Bélgica, España, Finlandia, Francia, Grecia, Holanda, Italia, Portugal, Suecia y Suiza.

\section{Designador de blancos electro-óptico de cuarta generación (Joint Airborne NavigatioN and Attack -JOANNA-).}

El Programa para el desarrollo de un demostrador tecnológico primero y de un equipo operativo posterior, de un designador de blancos electro-óptico de cuarta generación, que sustituya a los actualmente en servicio en las Fuerzas Aéreas Europeas, se inició en 1999 por Francia y Gran Bretaña, invitando posteriormente a participar a España e Italia que están negociando en estos momentos las condiciones para su entrada en este Programa de Colaboración Europeo.

La particularidad que presenta este Programa en lo que se refiere a los aspectos técnicos de la colaøoración española, estriba en que España está ofreciendo las capacidades que tiene el Ejército del Aire de integración de armamento y equipos en sus Sistemas de Armas aéreos en su 
Centro de Experimentación en Vuelo, ello con independencia de la participación industrial española que corresponda.

Entre las mejoras que se quieren obtener respecto a los designadores actualmente en servicio se pueden mencionar las siguientes:

- Aumento del alcance de manera que se pueda actuar fuera del alcance de las armas del adversario («stand-off»).

- Aumento de la resolución para mejorar la capacidad de identificación y poder de esta manera reducir los daños colaterales.

- Integración de un detector óptico avanzado de gran estabilidad.

- Desarrollo de algoritmos para la detección e identificación automática de los blancos, suplementado por la utilización de tecnología electro-óptica activa.

- Adquisición y seguimiento de blancos múltiples.

\section{Alliance Ground Surveillance-AGS-}

En marzo de 1993, en el seno de la OTAN se identificó el requisito operativo para dotar a esta organización de un sistema integrado en plataformas aéreas para el reconocimiento y vigilancia en cualquier condición atmosférica, día y noche, situado fuera del alcance de las armas del adversario ("stand-off"). El sistema permitirá a las unidades de combate de la OTAN vigilar un amplio espacio terrestre desde una distancia considerable, seleccionar los blancos del adversario y dirigir el fuego contra ellos.

A pesar de que este programa, considerado como un Programa de Colaboración Transatlántica, tiene una alta prioridad dentro de la OTAN, el comienzo de su desarrollo se está retrasando debido a los diferentes puntos de vista existentes entre las naciones participantes en lo que se refiere a la tecnología a emplear y a la capacidad de compartirla entre todas ellas.

La propuesta inicial consistía en la utilización conjunta del sistema americano en servicio, el Sistema Conjunto de Vigilancia y Radar de Ataque de Blancos (Joint Surveillance and Target Attack Radar System -JSTARS-), propuesta que fue desechada entre otras razones por no presentar una solución de tecnología futura.

La siguiente propuesta fue el desarrollo de un nuevo sistema basado en el JSTARS, al que se incorporarían los últimos avances tecnológicos en los sensores de detección y en el procesamiento y transmisión de la información. El nuevo sistema se denominaría NATAR, «NATO Advanced Transatlantic Radar», y fue aceptado por Bélgica, Canadá, Dinamarca, Luxemburgo y Noruega; pero el resto de las naciones europeas participantes en el Programa, las de más nivel de desarrollo tecnológico, Alemania, España, Francia, Holanda e Italia (Gran Bretaña va a desarrollar 
Colaboración internacional en la industria aeroespacial...

su propio sistema), lo rechazaron por las limitaciones impuestas por los Estados Unidos a la transferencia de tecnología y dominio operativo del mismo por todas las naciones, proponiendo a su vez en contrapartida una solución europea a desarrollar por estos países denominada SOSTAR, «Stand-Off Surveillance and Target Acquisition Radar», para lo cual se ha constituido un consorcio industrial formado por las compañías EADS (DASA) de Alemania, INDRA de España, THALES de Francia, FOKKER SPACE de Holanda y FIAT de Italia.

No obstante, las conversaciones han continuado y se han hecho progresos importantes para llegar a una solución de consenso que permita disponer finalmente de un sistema conjunto.

El nuevo sistema se pretende que tenga unas características muy avanzadas, para lo que estará dotado de un radar de apertura sintética (Synthetic Aperture Radar -SAR-) de ultra alta resolución y apertura invertida (Inverse SAR), con la función de indicador de movimiento de blancos (Movement Target Indicator -MTI-) con resolución para grandes distancias, y con enlace de datos protegido contra perturbación electrónica. Esta tecnología permitirá la detección, determinación de la situación y seguimiento del movimiento de los blancos terrestres, distinguiendo el tipo de vehículos detectados ya sean de ruedas o remolques, si se trata de vehículos civiles o de tipo militar, e incluso si se trata de helicópteros, así como identificar las antenas de los sistemas radar de vigilancia y defensa aérea. El radar SAR, con antenas de barrido electrónico activo, proporcionará imágenes que permitan a los operadores del sistema, mediante su análisis, determinar cuáles son los blancos críticos dentro del campo de batalla, tales como puentes, aeropuertos, puertos, principales vías de comunicación y emplazamientos radar; por su parte, el modo MTI del radar presentará una serie de puntos de color que representan el movimiento, en tiempo casi real, de los blancos sobre la superficie. Mediante el análisis de estos datos y su correlación con otras fuentes de información, los operadores del sistema podrán determinar si los blancos móviles son vehículos civiles o amenazas potenciales, tales como un convoy de carros de combate o helicópteros volando a baja altura. La información obtenida se enviará por enlace de datos protegido a las estaciones de tierra para su diseminación a las unidades terrestres combatientes.

\section{Programa Europeo de Desarrollo Tecnológico (European Technology Acquisition Program -ETAP-)}

Cuando se encuentra cercana la entrada en servicio operativo del Eurofighter 2000, las naciones europeas ya han empezado ha plantearse su 
futura sustitución junto con la del resto de aviones de su generación. Esto que podía parecer un poco prematuro, no lo es en realidad dado el dilatado espacio de tiempo que hemos podido ver que transcurre desde que se empiezan a elaborar los requisitos operativos y se lleva a cabo el diseño y desarrollo del sistema, hasta que éste entra finalmente en servicio operativo.

De esta manera, en el seno del Grupo de Armamentos de la Europa Occidental (Western European Armaments Group -WEAG-), se creó en 1998 un Grupo de Trabajo denominado Futuro Avión de Combate (Future Combat Aircraft-FCA-), para formalizar de manera conjunta los requisitos de las naciones europeas para la sustitución de los aviones de combate actualmente en servicio. Por su parte, de manera independiente, Gran Bretaña había iniciado estudios similares en 1990 con un proyecto denominado Futuro Avión Ofensivo (Future Offensive Aircraft -FOA-), que fue modificado conceptualmente en 1997 dándole el nombre de Futuro Sistema Ofensivo Aéreo (Future Offensive Air System -FOAS-), al comprender el nuevo proyecto la posibilidad de integrar tanto aviones tripulados como no tripulados (Unmanned Combat Air Vehicle-UCAV-), y misiles de crucero lanzados desde plataformas aéreas. En la misma línea, Alemania había iniciado un programa similar denominado Futuro Sistema de Armas Aéreo (Future Airborne Weapon System -FAWS-).

Dado que España, Francia, Italia y Suecia tenían también planes para un programa de estas características, decidieron unir sus esfuerzos a los de Alemania y Gran Bretaña, iniciando un Programa de Colaboración Europeo de Demostración de Tecnologías que, eventualmente, conducirá al desarrollo de un Sistema como el mencionado.

A estos efectos, el pasado mes de noviembre, en Bruselas, firmaron un Memorandum de Entendimiento para el lanzamiento del «Programa Europeo de Desarrollo Tecnológico (European Technology Acquisition Program -ETAP-)». Entre los objetivos del programa está el desarrollo y demostración de tecnologías avanzadas que permitan reducir el riesgo y el coste de las futuras adquisiciones de Sistemas de Armas de combate aéreos, así como la mejora de los mismos en términos de flexibilidad, capacidad de destrucción, disponibilidad y supervivencia. Dentro de él se espera trabajar en un número de proyectos diferentes del orden de 25, entre los cuales uno de los más significativos es el que se refiere a la tecnología «stealth», para incrementar la capacidad de los sistemas aéreos de evitar ser detectados por los diversos sensores que posea el adversario, que es un área donde los americanos aventajan en mayor medida a los europeos. 
Colaboración internacional en la industria aeroespacial...

Este Programa se estima que constituye una buena oportunidad para la industria aerospacial europea de permanecer en el negocio de los aviones de combate.

\section{Conclusiones}

De lo expuesto hasta aquí, se deduce que el Ejército del Aire ha apostado decididamente por la cooperación internacional, esencialmente la europea, para el desarrollo de los programas con que se dota de los medios que requiere para el desarrollo de su actividad operativa que, para que pueda alcanzar el grado de eficacia requerido, está estrechamente vinculada al desarrollo científico y tecnológico. Esta colaboración permite, por otra parte, reducir el coste de los Sistemas de Armas haciéndolos más asequibles a los presupuestos nacionales, así como incrementar sustancialmente la interoperabilidad del Ejército del Aire con las Fuerzas Aéreas de los países aliados, mejorando de esta manera su eficacia conjunta en las operaciones, cada día más numerosas, en que intervengan como una coalición internacional.

Como ventaja adicional, las colaboraciones llevadas a cabo han permitido que el sector aerospacial de la industria nacional se pueda posicionar adecuadamente dentro de Europa y del mundo occidental, lo que tiene gran trascendencia en estos momentos en que se está llevando a cabo una reestructuración en profundidad de la misma

No hay duda de que la intensa colaboración que está teniendo lugar en los países europeos en el área de los programas de Defensa es un factor esencial que contribuye al proceso de consolidación de la Identidad Europea. No obstante, parece lógico que estas colaboraciones se amplíen en el futuro pasando a tener un carácter transatlántico con la participación de los Estados Unidos, aunque esto requiere todavía cierto tiempo para que se vayan eliminando las barreras existentes que impiden trabajar en un plano de igualdad, sin reservas en cuanto al conocimiento mutuo y la transferencia de tecnología y al empleo sin restricciones de los medios adquiridos en uno u otro lado del Atlántico.

\section{Bibliografía}

Libro del 50 aniversario de la OTAN.

Décima Sesión Europea de los Responsables de Armamento.

Carta de Intenciones relativa a las medidas para facilitar la reestructuración de la Industria de Defensa Europea. 


\section{Fernando Mosquera Silvén}

Informe de la Comisión de Finanzas del Parlamento Francés sobre la financiación del Programa Europeo del avión de transporte militar, de 1994.

Diversos artículos de revistas y periódicos especializados (Air Force Magazine, Armed Forces Journal, Aviation Week \& Space Technology, Defense News, Flight International, Jane's Defence Weekly, Jane's International Defense Review, Military Technology, Revista de Aeronáutica y Astronáutica, etc). 